Gynäk. Rdsch. 1970;10:312

\title{
Ungewöhnliche Uterusperforation mit einem neuen
} Intrauterinpessar

H.

Lehfeldt

Department of Obstetrics and Gynecology of New York University School of Medicine, New York

Die Autoren berichten von einem 1966 eingeführten

in T-Form ge-stalteten IUP. In einer Vorstudie fanden sie eine geringe Ausstossungs-tendenz und kaum Nebeneffekte

der Schwangerschaftsverhütungseffektwar dagegen sehr schlecht. Durch Umwicklung mit 0,2 mm dickemKupferdraht konnte die Antikonzeption gesteigert werden (Tcu-200 Ta-tum).

Nachdem in Arbeit en von anderen Kliniken über insgesamt 4000Einlagen nie eine Perforation festgestellt werden konnte

berichten dieAutoren der vorliegenden Arbeit über zwei Perforationen im proximalenAnteil des Zervixkanals

wobei es zu keiner klinischen Symptomatikkam ausser einem leichten Irritationsgefühl. Der Nachweis konnte nurdurch die Hysterographie geführt werden.

B. Geiser

1 Originaltitel: Unusual perforation with a new intrauterine device. 